Biochimica et Biophysica Acta, 382 (1975) 22-26

(C) Elsevier Scientific Publishing Company, Amsterdam - Printed in The Netherlands

BBA 76871

\title{
BARRIER PROPERTIES OF LECITHIN/LYSOLECITHIN MIXTURES
}

\author{
J. G. MANDERSLOOT, F. C. REMAN, L. L. M. VAN DEENEN and J. DE GIER \\ Laboratory of Biochemistry, University of Utrecht, University Centre "De Uithof", Padualaan 8 , \\ Utrecht (The Netherlands)
}

(Received September 4th, 1974)

\section{SUMMARY}

Light scattering, birefringence and X-ray studies showed that liposomes, with lipid molecules orientated in bilayers, are formed from egg lecithin/lysolecithin mixtures up to $50 \mathrm{~mol} \%$ of lysolecithin; above this concentration much smaller mixed micelles are formed. Permeability studies demonstrated a dramatic increase in the permeability of the liposomes when the lyso concentration exceeds $22.5 \mathrm{~mol} \%$. X-ray studies indicated a significant decrease in bilayer thickness with increasing lysolecithin concentration. It is suggested that decreased interaction energy between the lipid molecules in the bilayer is responsible for the inability of the thin bilayers to act as an effective permeability barrier.

Besides diacyl phospholipids, minor quantities of lysophospholipids occur as normal constituents of many biomembranes [1]. The lysophospholipids in the membrane are involved in dynamic metabolic processes and the actual concentration seems to be under careful control of various enzyme systems achieving the formation, acylation and breakdown of the lyso compounds [2]. It is generally accepted that the concentration of lyso compounds has important implications for the stability of the interface [3]. Increased lysolecithin concentrations favour conditions for cell fusion [4] and excess of exogenously added lysolecithin causes lysis. Lysis of, for example, red cells has been regarded as primarily an interaction of the lysolecithin molecules with the lipid bilayer in the membrane core. Postulating a "wedge" mechanism for the penetrating lyso molecules, complete break up of the bimolecular leaflet into more or less spherical aggregates of molecules can be considered [3]. On the other hand the lytic process has also been described as being the consequence of more subtle changes in the bilayer orientation causing increase in cation permeability. The disturbance of the Donnan equilibrium then causes entry of water into the cell which bursts by osmotic force $[5,6]$. Experiments with artificial lipid membranes can be helpful in gaining information on the effects of lysolecithins in biomembranes.

Studies on black lipid membranes supported the view that the lytic molecules interfere with the lipid bilayer. In comparative experiments in which lysolecithins or lytic short chain lecithins were added to red cells and to black lipid membranes pre- 
pared of red cell total lipids, good correlations could be observed in the kinetics of the red cell lysis and of the break of the black lipid membranes. Below concentrations roughly corresponding to the critical micelle concentration [14] (CMC) the rate of both processes was found to be strongly dependent on the concentration, but above the CMC the dependency was much less [7]. This strongly suggests that the membrane-attacking lytic agent is the monomeric form of the lyso compound and not the micelle [8]. Black lipid membranes prepared with decane solutions containing mixtures of lecithin and lysolecithin showed that stable bilayer films could be obtained from mixtures containing up to $20 \mathrm{~mol} \%$ of lysolecithin in the film forming solution. The films prepared of solutions containing more than $2 \mathrm{~mol} \%$ lysolecithin demonstrated a marked decreased electrical resistance [9]. Although the actual concentrations of lysolecithin in the black lipid membranes is unknown, and the presence of decane is a complicating factor in this system, the results suggest that long before the bilayer falls apart into spherical aggregates, changes in permeability occur.

Studies on liposome systems confirm remarkable stability of lipid bilayers in the presence of relative high concentrations of lysolecithin. Fig. 1 shows the absorbance of dispersions in $150 \mathrm{mM} \mathrm{KCl}$ of mixtures of egg lecithin and lysolecithin

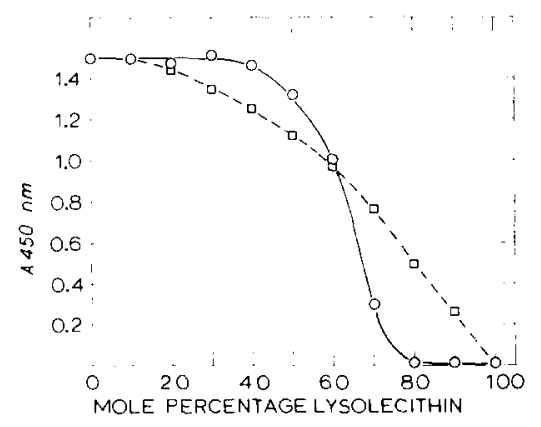

Fig. 1. Turbidity of lecithin/lysolecithin mixtures dispersed in a water phase. $\bigcirc-\bigcirc$, mixing was done before addition to the water phase. $\square-\square$, lecithin and lysolecithin were added separately to the water phase. Samples of lecithin or lecithin/lysolecithin mixtures were pipetted as chloroform solutions into test tubes and blown to dryness by a stream of $\mathrm{N}_{2}$. After addition of $1 \mathrm{ml}$ of KCl, $150 \mathrm{mM}$, and 1 glass bead liposomes were prepared by gentle shaking. Subsequently the dispersion was diluted with $5 \mathrm{ml} \mathrm{KCl}, 150 \mathrm{mM}$. When lysolecithin was added separately, the lyso compound was solubilized in this $5 \mathrm{ml}$ of diluting solvent. The total amount in each tube was $5 \mu$ mol phospholipid. Absorbances were measured at $450 \mathrm{~nm}$.

prepared from this lecithin. In these experiments the total phospholipid concentration is kept constant, but the ratio and the way of mixing is varied. When the diacyl and lyso compound are mixed in chloroform and the homogeneous mixture after evaporation of chloroform is dispersed in the water phase, mixed liposomes are formed up to a concentration of about $50 \mathrm{~mol} \%$ lysolecithin. A similar pattern has been reported for the system of brain gangliosides plus egg lecithin [17]. X-ray analyses demonstrated that around $50 \mathrm{~mol} \%$ lysolecithin the X-ray pattern is complicated, but that below this concentration there is solely a lamellar phase. Microscopic examination of the lipid-water dispersions demonstrated at all these concentrations particles with bire- 
fringence characteristic for liposomes. The existence of stable lipid bilayers up to high concentrations of lysolecithin was also noticed in recent differential scanning calorimetric and freeze fracture electron microscopic studies [10] on mixtures of dipalmitoyl and monopalmitoyl glycerylphosphorylcholine. The rapid drop in absorbance above $50 \mathrm{~mol} \%$ of the lyso compound can be explained by the "wedge" function of the lyso molecules and the formation of small spherical aggregates is very likely. Fig. 1 also shows the effect when the lecithin is allowed to form liposomes first and lysolecithin is added subsequently. The completely different absorbances, measured after $1 \mathrm{~h}$ equilibration, suggest that the penetration of the lysolecithin, into the lecithin bilayers is only limited. Even when added in large excess, the lysolecithin is unable to "solubilize" the lecithin liposomes. As a possible explanation we suppose that, by disruption of only a few of the liposomal bilayers, mixed micelles are formed causing a rapid decrease of the monomeric concentration, which brings the penetration process to a fading end. In agreement with these results we noticed that lysolecithin added in large excess to sonicated liposomes with ${ }^{86} \mathrm{Rb}^{+}$trapped inside caused no rapid release of the radioactive ion but only a limited increase in the rate of leak extending over a long period of time [6]. Based on these observations we think that the liposome system is not very suitable to study the kinetics of the lytic process. However, the homogeneous (chloroform) mixed systems allow the permeability properties of bilayers of mixtures of mono- and diacyl phospholipids to be studied. Fig. 2 shows

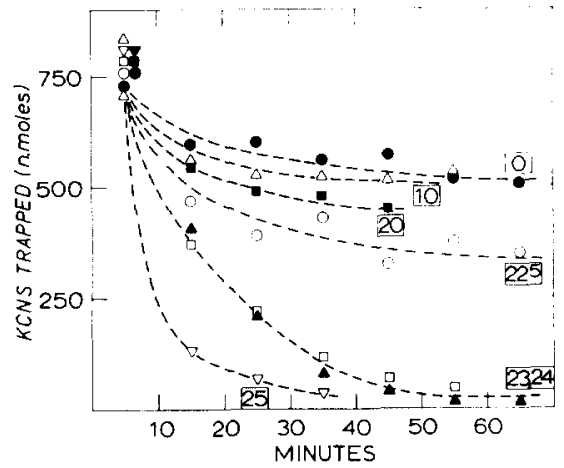

Fig. 2. Trapping of $\mathbf{K}^{+}$ions inside liposomes prepared from lecithin/lysolecithin mixtures. Liposomes were prepared by dispersing dry lecithin containing $4 \mathrm{~mol} \%$ phosphatidic acid and variable concentrations of lysolecithin into $150 \mathrm{mM}$ KCNS. $1 \mathrm{ml}$ samples of the liposome dispersions containing $20 \mu \mathrm{M}$ of phospholipid were dialysed against $150 \mathrm{mM} \mathrm{MgSO}_{4}$ at $13{ }^{\circ} \mathrm{C}$. The dialysing solution was refreshed after $2 \frac{1}{2}, 10,20,30,40,50$ and $60 \mathrm{~min}$. The trap inside the liposomes was measured at different time intervals by bringing $100 \mu \mathrm{l}$ of the liposome dispersion into $5 \mathrm{mI}$ of $150 \mathrm{mM} \mathrm{MgSO}_{4}$ containing $10 \mathrm{mM}$ Tris $\cdot \mathrm{HCl}, \mathrm{pH} 7.4$. The $\mathrm{K}^{+}$inside the structures expressed as nmol of $\mathrm{K}^{+}$per $\mu$ mol of phospholipid was calculated from the increase in $\mathrm{K}^{+}$activity upon addition of $100 \mu \mathrm{l}$ Triton $\mathrm{X}-100$. This $\mathrm{K}^{+}$activity was measured with a $\mathrm{K}^{+}$sensitive glass electrode as described earlier [14].

the ability of the mixed liposomes to trap KCNS. Up to $22.5 \mathrm{~mol} \%$ the lysolecithin has only little effect on the permeability behaviour of the liposomes. This is in agreement with a recent report on glucose leak from lysolecithin-containing liposomes [11]. However, at $22.5 \mathrm{~mol} \%$ there is a rapid increase in permeability and the $\mathrm{K}^{+}$ions originally enclosed by the liposome structures can easily be removed by dialysis. 
In order to explain this increase in permeability the results of X-ray analyses on these liposomes may be relvant. Fig. 3 shows that with increasing lysolecithin concentration there is a significant decrease in the bilayer thickness. When we consider that the polar groups (phosphorylcholine) remain the same then the decrease in thickness is

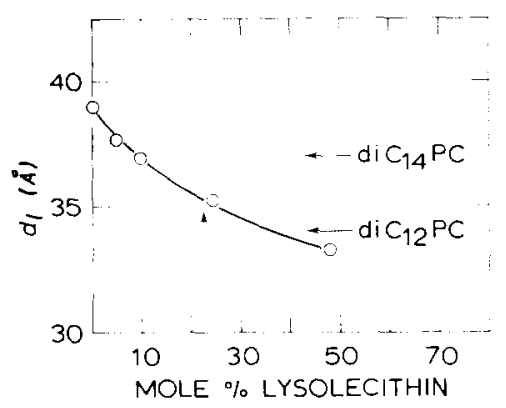

Fig. 3. Thickness of the lecithin/lysolecithin bilayer as a function of the lysolecithin-concentration. Lecithin and lysolecithin were mixed in varying amounts and $4 \mathrm{~mol} \%$ of phosphatidic acid was added. The chloroform/methanol solutions were taken to dryness and stored over $\mathrm{P}_{2} \mathrm{O}_{3}$ for $24 \mathrm{~h}$ in vacuo. Small amounts, $20-30 \mathrm{mg}$, were weighed out accurately and distilled water was added to give a final concentration of the water of $40-50 \%$ (determined by weighing the samples again). The lipid/water mixtures were allowed to equilibrate for $48-72 \mathrm{~h}$, after which the X-ray experiments [16] were carried out.

due solely to a decrease in the paraffin part of the bilayer. Assuming a thickness of about $16-18 \AA$ for the polar parts of the double layers the thickness of the paraffin layer of pure egg lecithin bilayers is about $21-23 \AA$. With $50 \mathrm{~mol} \%$ of lysolecithin this value is reduced to $15-17 \AA$, a decrease of about $30 \%$. For reasons of comparison, the bilayer thickness of the bilayers in pure dimyristoyl lecithin and dilauroyl lecithinwater systems are also indicated in Fig. 3. We have noticed that liposomes of dimyristoyl lecithin are capable of trapping ions both below and above the liquid-gel transition temperature, but that dilauroyl lecithin liposomes are completely permeable and loose their enclosed ions rapidly during dialysis. The thickness of the lipid bilayers in liposomes of egg lecithin with $22.5 \mathrm{~mol} \%$ of lysolecithin (Fig. 3) is well below the thickness of the dimyristoyl lecithin. Therefore, although the mechanism of ion penetration is not clear, we conclude tentatively that the thickness of the paraffin core is critical for the bilayer to act as an effective permeability barrier. It can be suggested that, with decreasing thickness of the paraffin core, there is a decrease in interaction energy between the lipid molecules, and that below a critical value a stable association of the lipid molecules in the bilayer is no longer warranted [12, 13].

These observations may also have implications with respect to the haemolytic process. When we assume that these model experiments can be extrapolated to the erythrocyte membrane, they support the hypothesis than an important stage in the lysis by lysolecithin is a change in cation permeability, inducing swelling of the cell and leading to a progressive osmotic breakdown of the membrane.

\section{ACKNOWLEDGEMENT}

One of us, F.C.R., wishes to express his gratitude to Dr. V. Luzzati of the 
Centre Génétique Moléculaire du C.N.R.S. at Gif-sur-Yvette for his permission to carry out the X-ray experiments in this laboratory, and to Dr. A. Tardieu for her collaboration in the experiments and many stimulating discussions.

\section{REFERENCES}

I Ansell, G. B., Dawson, R. M. C. and Hawthorne, J. N. (1973) Form and Function of Phospholipids, pp. 444-467, Elsevier, Amsterdam

2 Van den Bosch, H. (1974) Annu. Rev. Biochem. 43, 243-278

3 Haydon, D. A. and Taylor, J. (1963) J. Theoret. Biol. 4, 281-296

4 Lucy, J. A. (1970) Nature 227, 815-817

5 Rideal, E. and Taylor, F. H. (1958) Proc. Roy. Soc. B. 148, 450 464

6 Reman, F. C. (1971) Thesis, Utrecht, The Netherlands

7 Reman, F. C., Demel, R. A., de Gier, J., van Deenen, L. L. M., Eibl, H. and Westphal, O. (1969) Chem. Phys. Lipids 3, 221-233

8 Jung, F. (1959) Acta Biol. Med. Ger. 2, 481-495

9 Van Zutphen, H. and van Deenen, L. L. M. (1967) Chem. Phys. Lipids 1, 389-391

10 Klopfenstein, W. E., de Kruyff, B., Verkleij, A. J., Demel, R. A. and van Deenen, L. L. M. (1974) Chem. Phys. Lipids, in the press

11 Smolen, J. E. and Shohet, S. B. (1974) J. Lipid Res. 15, 273-280

12 Hauser, H. and Barratt, M. D. (1973) Biochem. Biophys. Res. Commun. 53, 399-405

13 Phillips, M. C., Williams, R. M. and Chapman, D. (1969) Chem. Phys. Lipids 3, 234-244

14 Hayashi, M., Okasaki, M. and Hara, I. (1972) in Chemie, Physikalische Chemie und Anwendungstechnik der Grenzflächenaktiven Stoffe, Band II, Sektion B, pp. 361-370, Carl Hansen Verlag, München

15 Haest, C. W. M., de Gier, J., van Es. G. A., Verkleij, A. J. and van Deenen, L. L. M. (1972) Biochim. Biophys. Acta 288, 43-53

16 Gulik-Krzywicki, T., Rivas, E. and Luzzati, V. (1967) J. Mol. Biol. 27, 303-322

17 Hill, M. W. and Lester, R. (1972) Biochim. Biophys. Acta 282, 18-30 